\title{
Antibacterial Property of Hanguana Malayana (Bakong) Crude Leaf Ethanolic Extract Against Staphylococcus Aureus
}

\author{
Vandon T. Borela ${ }^{1}$, Jan Arianne B. Urbano ${ }^{2}$, Andrei Joshua A. Tayag ${ }^{2}$, \\ Myco G. Teresa ${ }^{2}$ \\ *Correspondence: borelavandon89@gmail.com \\ ${ }^{1}$ Teacher, Parang High School, Philippines \\ ${ }^{2}$ Students, Parang High School, Philippines
}

\begin{abstract}
Hanguana Malayana, known as Bakong plant is an invasive macrophytic plant found in few regions of the Philippines. Due to its mode of propagation, it occupies large spaces and it invades the space that's allotted for the crops of local farmers. Hence, the researchers investigate this plant for beneficial uses. In this study, the effect of the different concentrations of H. malayana crude ethanolic leaf extract on Saureus is evaluated on the basis of the study of Ooh and company (2014) stating that the plant is a potential resources of bioactive phytoconstituents. This research concluded that the Hanguana malayana crude leaf ethanolic extract has an antibacterial property against Staphylococcus aureus.
\end{abstract}

Keywords: Hanguana Malayana, Staphylococcus Aureus, Inhibition Zone

Received : November 1, 2020

Revised: December 25, 2020

Accepted: January 6, 2021

\section{Introduction}

Hanguana Malayana is a wetland macrophyte native in the Philippines and is localized in Laguna de Cagayan, where it is formerly known as a pest that thrives in wetlands and terrestrial habitats until the plant is known to be a good source of fiber.

A macrophyte is an sea-going plant that develops in or close water and is either emanant, submergent, or coasting. Most macrophytes develop actually within the wild; but, a few are developed and devoured by people as plant nourishment. Around the world, different wetland macrophytes are utilized customarily to treat human infections. Wetland macrophytes are a potential characteristic asset for the revelation of therapeutically-relevant normal items (Chambers et al., 2007; Li et al., 2013). To date, the health-promoting phytochemicals and bioactivities of numerous macrophytes are underexplored. Wetlands give a one of a kind living space for a few restorative plants. In Muriyad, it is said that Neighborhood individuals utilize a wide assortment of wetland/wetland-associated plants as fixings of conventional home grown therapeutic arrangements.

Customarily, H. malayana is utilized remotely to treat fever. Agreeing to the phytochemical screening of Ooh et al, Hanguana Malayana contains the nearness and concentration of four sorts of HBAs, to be specific gallic acid (GA), p-HBA, vanillic acid (VA) and protocatechuic acid (PCA). Phenolic constituents of plants are a conspicuous source of health-promoting phytochemicals. Plant phenolic compounds are basically differing and can be separated into diverse classes, counting hydroxybenzoic acids (HBAs), hydroxycinnamic acids, and flavonoids. 
Gallic acid could be a well-known characteristic antioxidant that's essentially a auxiliary polyphenolic metabolite (Alcalde et al.,2019; Martini et al., 2020). Gallic acid may be a exceptionally vital common antioxidant tea detailing, known as an Ayurvedic herb. Agreeing to ponders, Polyphenols decreased chance of rate of numerous cancers in people with the eat less wealthy in natural products and vegetables. Plant-derived phenolic compound is utilized in cancer chemoprevention. Gallic acid has been appeared to display natural action, counting anti-cancer. Gallic acid has moreover been detailed to show anti-invasive and anti-metastatic exercises in different cancer cells. It may be a potential preventive and helpful specialist against gastric cancer metastasis. Another ponder in human glioma cells has indicated that gallic acid hinders cell expansion and altogether diminishes cell reasonability, attack and tube arrangement. p-HBA or p-Hydroxybenzoic moreover known as 4-Hydroxybenzoic Acid may be a monohydroxybenzoic acid, a phenolic subordinate of benzoic acid (Farhoosh et al., 2016; Farhoosh et al., 2016). It is basically known as the premise for the planning of its esters, known as parabens. Parabens are a lesson of broadly utilized additives in corrective and pharmaceutical items.

Vanillic acid may be a phenolic acid found in a few shapes of vanilla and many other plant extricates. It could be a flavoring and scent operator that produces a charming, velvety odor. It is the intermediate product within the two-step bioconversion of ferulic acid to vanillin. Vanillic acid, which could be a chlorogenic acid, is an oxidized shape of vanillin. It is additionally an middle of the road within the generation of vanillin from ferulic acid. Vanillic acid may be a metabolic byproduct of caffeic acid and is frequently found within the pee of people who have expended coffee, chocolate, tea and vanilla-flavored confectionary. Vanillic acid specifically and particularly hinders 5'nucleotidase movement.

Protocatechuic acid could be a dihydroxybenzoic acid, a type of phenolic acid. It may be a major metabolite of antioxidant polyphenols found in green tea. It has blended impacts on ordinary and cancer cells in in vitro and in vivo thinks about. Protocatechuic acid (PCA) is antioxidant and anti-inflammatory. PCA has been detailed to initiate apoptosis of human leukemia cells, as well as harmful HSG1 cells taken from human verbal cavities, but PCA was found to have blended impacts on TPA-induced mouse skin tumors (Hyer et al., 2000; Cheok et al., 2003; Ong et al., 2012). Depending on the sum of PCA and the time before application, PCA may diminish or upgrade tumor development. So also, PCA was detailed to extend multiplication and hinder apoptosis of neural stem cells.

Essentially, PCA was detailed to extend multiplication and restrain apoptosis of neural stem cells [20]. In an in vitro demonstrate utilizing HL-60leukemia cells, protocatechuic acid appeared an antigenotoxic impact and tumoricidal activity. Hanguana Malayana contains the concentrations for six hydroxycinnamic acids within the plant extricates, specifically, p-CA, ferulic acid (FA), chlorogenic acid (ChA), caffeic acid (CFA), sinapic acid (SNA) and syringic acid (SA).

Coumaric acid could be a hydroxyl subordinate of cinnamic acid and actually happens in three isomers (ortho-, meta- and para-); p-coumaric acid is one of the foremost commonly happening isomer in nature. p-Coumaric acid (4-hydroxycinnamic acid) may be a phenolic acid that serves as a antecedent of other phenolic compounds, and exists either in free or conjugated shape in plants. Conjugates of p-coumaric acid have been broadly examined in later a long time due to their bioactivities. In this audit, the event, bioavailability and bio accessibility of p-coumaric acid and its conjugates with mono-, oligo- and polysaccharides, alkyl alcohols, natural acids, amine and lignin are examined. Their organic exercises, counting antioxidant, anti-cancer, antimicrobial, antivirus, anti-inflammatory, antiplatelet 
conglomeration, anxiolytic, antipyretic, pain relieving, and anti-arthritis exercises, and their mitigatory impacts against diabetes, weight, hyperlipidemia and gout are compared.

Add up to demonstrate from various considers appears that conjugation of p-coumaric acid hugely fortifies its common works out; in any case, the tall natural development but moo maintenance of its conjugates remains a flabbergast. Concurring to considers, Ferulic acid may well be a hydroxycinnamic acid found in cell dividers of plants such as rice and oats and the seeds of apples and oranges, where it plays a key portion inside the plants' affirmation and self-preservation. Ferulic acid is illustrated to direct developing handle by diminishing the impacts of hurting free radicals on the skin, it is said that it additionally guarantees skin from sun hurt. Another great thing approximately it is it appears synergistic affect adjacent other cancer avoidance operators like Vitamin $\mathrm{C}$ and $\mathrm{E}$.

Total prove from numerous ponders shows that conjugation of p-coumaric acid enormously reinforces its natural exercises; in any case, the tall organic movement but moo retention of its conjugates remains a astound. Agreeing to ponders, Ferulic acid could be a hydroxycinnamic acid found in cell dividers of plants such as rice and oats and the seeds of apples and oranges, where it plays a key part within the plants' assurance and selfpreservation. Ferulic acid is demonstrated to moderate maturing handle by decreasing the impacts of harming free radicals on the skin, it is said that it moreover ensures skin from sun harm. Another good thing about it is it shows synergistic impact nearby other cancer prevention agents like Vitamin $\mathrm{C}$ and $\mathrm{E}$.

\section{Methods}

Hanguana malayana was obtained from Sta. Teresita, Cagayan. The plant was brought to the University of the Philippines for its identification. Leaf samples of Hanguana malayana was air dried at room temperature for about a month. The dried leaf samples was powdered using mechanical blender. The powdered sample of H.malayana was macerated in $95 \%$ ethyl alcohol in jar for three days, and then it was filtered using filter paper. The filtrate evaporated and concentrated using a hot plate at 40 degree Celsius and in vacuo.

Two experimental treatments was made: $100 \%$ and $50 \%$, after the filtrate was concentrated it was weighed using an analytical balance and it was dissolved in distilled water until it is saturated. Further, the percent extraction yield is obtained using the formula as per Terblanche and company (2017). Percent extraction yield $=$ [amount $(\mathrm{g})$ of dried crude extract obtained)/(amount (g)of finelygrounded plant material used] x 100

Mueller Hinton Agar was cooked according to the manufacturer's label and then it was sterilized using a pressure cooker for 15 PSI for 15 minutes and then it was transferred to 12 petri dishes. Pour an amount that is enough to cover the bottom of the petri dish, leave it for a while to let it hardened.

Bacteria was given to the researchers in a vial. The researchers prepared the nutrient broth according to the manufacturer's label then it was sterilized using a pressure cooker for 15 PSI for 15 minutes. The bacteria was transferred to the broth using a sterile wooden applicator stick, aseptically. It was placed in a sterile storage box for 24 hours to let the bacteria grow. The bacteria was transferred to the working culture plates using a wooden applicator stick, following the Kirby-Bauer Disk Diffusion Method.

Filter discs was prepared using a paper punch. It was sterilized using a pressure cooker for 15 PSI for 15 minutes. The researchers used 12 petri dishes. 6 for the $100 \%$ extract and the other 6 for the $50 \%$ treatment. 
There are 12 filter discs (whatman filter paper no.1) all in all, 6 for the $100 \%$ and 6 for the $50 \%$. Using a syringe, $0.02 \mathrm{ml}$ of each treatment will be injected to The filter discs and will be dried for a couple of minutes. The treated filter discs was placed on working culture plates aseptically using a tweezer. It was sealed using a masking tape and was labeled to avoid mistakes. It was placed in a clean storage box or cabinet at room temperature and was observed for about two to three days to see the inhibition zone that forms around the filter discs. The inhibition zone was measured using a ruler. The radius or the diameter Of the inhibition zone was recorded. The antibacterial property of Hanguana Malayana was based on the size of the inhibition zone.

\section{Results and Discussion}

Table 1. Size of Inhibition zone

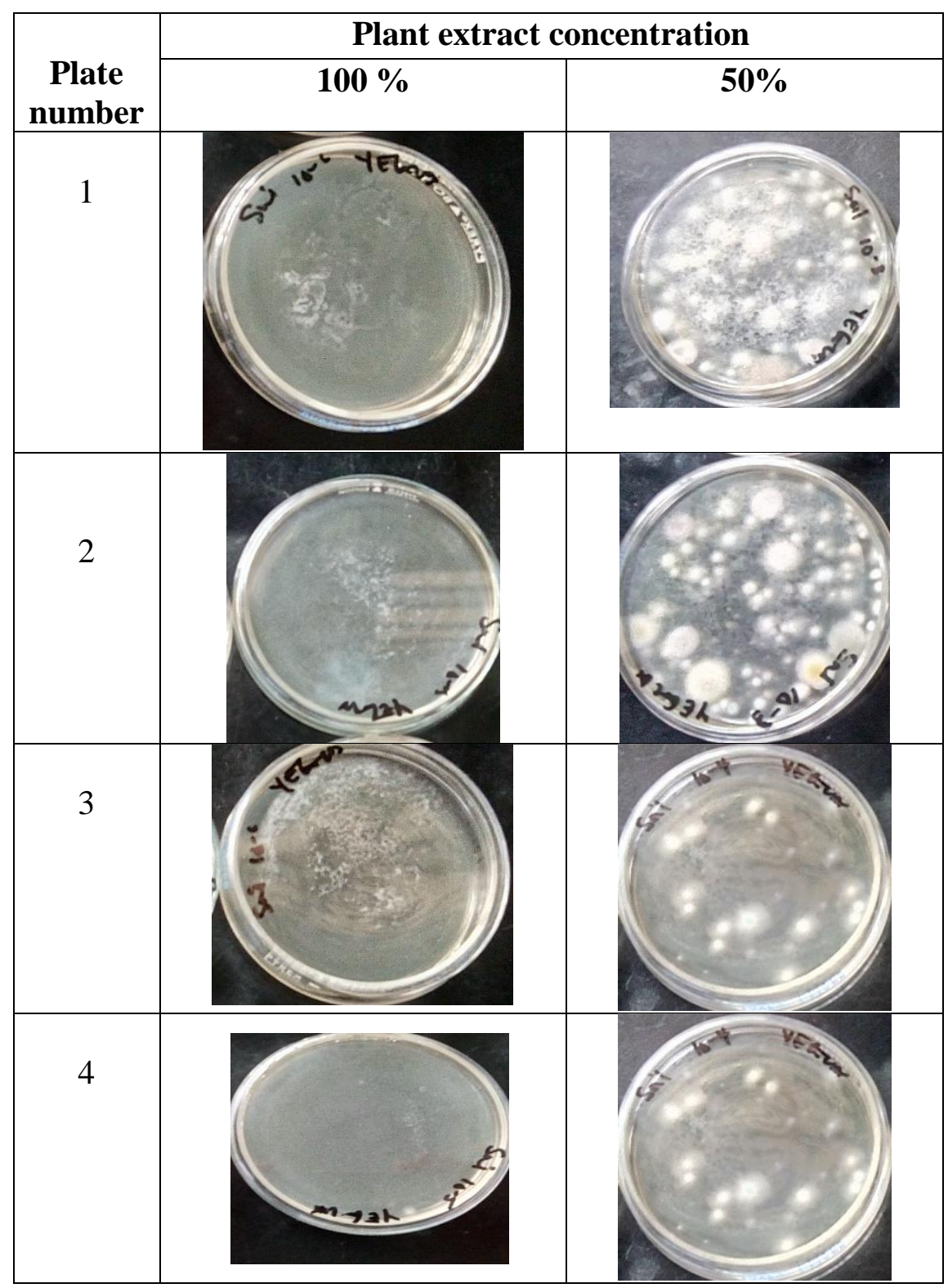

Table 1 shows the size of the inhibition zone. Based from the data gathered by the researcher, Table 1 shows the effect of the extract of the plant. Using the $50 \%$ of plant extract of $H$. malayana the $S$. aureus doesn't inhibit because it is observable on the colony of the S.aureus present on the four replicate. While on $100 \%$ plant extract of $H$. malayana, from plate 1 to 3

Copyright (C) 2021, International Journal Papier Advance and Scientific Review, Under the license CC BY-SA 4.0

DOI: https://doi.org/10.47667/ijpasr.v2i1.25 
there less number of colony while at plate 4 there are less number of colony. In this study, it was only proven to possess antibacterial property against gram-positive bacteria, Staphylococcus aureus. It is not yet known to be effective against gram-negative bacteria like Escherichia Coli. In the future studies, the efficacy of antibacterial property of Hanguana malayana on any type of bacterias should be evaluated to further know the ability of the plant.

\section{Conclusion}

Based from the data collected, the researcher concluded that using the $100 \%$ of Hanguana malayana inhibit the growth of Staphylococcus aureus even the researcher did not able to measure the size of the inhibition zone due to the effect of the plant extract. The bioactive compounds exhibits synergistic effect that made it have an effective antibacterial property against Staphylococcus aureus.

\section{REFERENCES}

Alcalde, B., Granados, M., \& Saurina, J. (2019). Exploring the antioxidant features of polyphenols by spectroscopic and electrochemical methods. Antioxidants, 8(11), 523.

Chambers, P. A., Lacoul, P., Murphy, K. J., \& Thomaz, S. M. (2007). Global diversity of aquatic macrophytes in freshwater. In Freshwater animal diversity assessment (pp. 9-26). Springer, Dordrecht.

Cheok, M. H., Yang, W., Pui, C. H., Downing, J. R., Cheng, C., Naeve, C. W., ... \& Evans, W. E. (2003). Treatment-specific changes in gene expression discriminate in vivo drug response in human leukemia cells. Nature genetics, 34(1), 85-90.

Farhoosh, R., Johnny, S., Asnaashari, M., Molaahmadibahraseman, N., \& Sharif, A. (2016). Structure-antioxidant activity relationships of o-hydroxyl, o-methoxy, and alkyl ester derivatives of p-hydroxybenzoic acid. Food chemistry, 194, 128-134.

Farhoosh, R., Sharif, A., Asnaashari, M., Johnny, S., \& Molaahmadibahraseman, N. (2016). Temperature-dependent mechanism of antioxidant activity of o-hydroxyl, omethoxy, and alkyl ester derivatives of p-hydroxybenzoic acid in fish oil. Journal of the American Oil Chemists' Society, 93(4), 555-567.

Hyer, M. L., Voelkel-Johnson, C., Rubinchik, S., Dong, J. Y., \& Norris, J. S. (2000). Intracellular Fas ligand expression causes Fas-mediated apoptosis in human prostate cancer cells resistant to monoclonal antibody-induced apoptosis. Molecular Therapy, 2(4), 348-358.

Li, W., Cao, T., Ni, L., Zhang, X., Zhu, G., \& Xie, P. (2013). Effects of water depth on carbon, nitrogen and phosphorus stoichiometry of five submersed macrophytes in an in situ experiment. Ecological Engineering, 61, 358-365.

Martini, S., Tagliazucchi, D., Minelli, G., \& Fiego, D. P. L. (2020). Influence of linseed and antioxidant-rich diets in pig nutrition on lipid oxidation during cooking and in vitro digestion of pork. Food Research International, 137, 109528.

Ong, Y. H., Lim, M., \& Liu, Q. (2012). Comparison of principal component analysis and biochemical component analysis in Raman spectroscopy for the discrimination of apoptosis and necrosis in K562 leukemia cells. Optics express, 20(20), 2215822171. 